\title{
R Package imputeTestbench to Compare Imputation Methods for Univariate Time Series
}

\author{
by Marcus W Beck, Neeraj Bokde, Gualberto Asencio-Cortés, and Kishore Kulat
}

\begin{abstract}
Missing observations are common in time series data and several methods are available to impute these values prior to analysis. Variation in statistical characteristics of univariate time series can have a profound effect on characteristics of missing observations and, therefore, the accuracy of different imputation methods. The imputeTestbench package can be used to compare the prediction accuracy of different methods as related to the amount and type of missing data for a user-supplied dataset. Missing data are simulated by removing observations completely at random or in blocks of different sizes depending on characteristics of the data. Several imputation algorithms are included with the package that vary from simple replacement with means to more complex interpolation methods. The testbench is not limited to the default functions and users can add or remove methods as needed. Plotting functions also allow comparative visualization of the behavior and effectiveness of different algorithms. We present example applications that demonstrate how the package can be used to understand differences in prediction accuracy between methods as affected by characteristics of a dataset and the nature of missing data.
\end{abstract}

\section{Introduction}

Univariate time series data provide valuable information that forms the basis of analysis across several disciplines. The most notable characteristic of time series data is time-dependent correlations between observations such that the likelihood of observing a single value depends on the values of past or future observations (Shumway and Stoffer, 2011; Box et al., 2015). This precludes the use of most conventional statistical methods that require independence of observations as a fundamental assumption prior to analysis. However, unique methods have been developed that account for and leverage the statistical characteristics of time series data to provide insight beyond those provided by more conventional techniques. For example, additive or multiplicative models can be used to separate a univariate time series data into unique components with different structures (e.g., Gould et al., 2008), whereas multiple methods for signal processing seek to isolate dominant components that are independent of random variation in the observed data (e.g., Mendel, 2000).

Many analysis methods for time series require complete observations at each time step across the period of interest. Complete datasets rarely exist such that missing observations are often defining characteristics of time series data (e.g., Gomez and Maravall, 1994; Honaker and King, 2010). Missing observations can occur for several reasons, although the type and amount of missing values depend on characteristics of the data (Schafer, 1997; Schafer and Graham, 2002). For example, a time series with no serial dependence between observations is more likely to have missing observations that are completely random (Rubin, 1976). A more common scenario for time series data is missing observations in 'chunks' or data missing in sequence for a period of time (Schafer, 1997; Donders et al., 2006). The occurrence of missing chunks relates directly to the correlation structure of time series data, as in cases when experiments are not continuously maintained, remote data fail to transmit from the source to the user, or funding mechanisms for monitoring programs are discontinuous. As such, the accuracy of imputation methods can vary considerably depending on characteristics of the dataset (Yozgatligil et al., 2013). Similar to the application of analysis methods that use time-dependent information, imputation methods must also faithfully reconstruct missing observations in this context.

Identifying an appropriate imputation method is often the first step towards more formal time series analysis. Different imputation methods will have differing precision in reproducing missing values, where precision will depend on how much data are missing and how the data are missing (i.e., individual observations missing at random or data missing in continuous chunks). The characteristics of the dataset will also influence imputation precision between methods. An expectation is that imputation methods that leverage characteristics of the dataset to predict missing values will perform better than more naïve methods, if indeed there is sufficient temporal structure. Accordingly, choosing an appropriate imputation method can be facilitated by using a standardized method of comparison. A simple approach for method comparison is to evaluate prediction accuracy from imputed values after removing observations from a test dataset, where the test dataset should have characteristics similar to the one requiring imputation. For example, Zhu et al. (2011) proposed a kernel-based iterative estimation method for missing data and compared the approach to other conventional frequency 


\begin{tabular}{lcccc}
\hline \multirow{2}{*}{ Method names } & \multicolumn{2}{c}{$\mathbf{1 0 \%}$} & \multicolumn{2}{c}{$\mathbf{8 0 \%}$} \\
& $T$ & $V$ & $T$ & $V$ \\
\hline Mixing & 8 & 0.085 & 20 & 1.53 \\
Poly & 10 & 0.103 & 25 & 2.11 \\
RBF & 11 & 0.107 & 29 & 2.86 \\
Normal & 14 & 0.121 & 30 & 3.01 \\
FE & 13 & 0.117 & 29 & 2.59 \\
\hline
\end{tabular}

Table 1: An approach for comparing imputation methods. Methods in the left column are compared by varying the amount of missing data $(10 \%$ and $80 \%)$ in a complete dataset and number of iterations ( $T$ ) for which the missing data are removed at random. $V$ is the mean RMSE of the imputed values. Reproduced from Zhu et al. (2011).

estimators. The methods were compared by simulating different amounts of missing data, predicting the missing values with each method, and then comparing the predictions to the actual data that were removed. Table 1 reproduces the results, where the rows show root-mean squared error (RMSE) between observed and predicted data for each imputation method after removing and predicting $10 \%$ and $80 \%$ of the complete dataset. Additional studies have used a similar workflow to compare the performance of imputation methods (Jörnsten et al., 2007; Li et al., 2015; Nguyen et al., 2013; Ran et al., 2015; Tak et al., 2016).

There are several challenges for adopting a standardized approach to compare imputation methods. An obvious concern is the amount of missing data, such that a complete gradient from very few to many observations should be evaluated with each method (Zhu et al., 2011). For example, one method may be superior for very few missing observations but perform poorly relative to other methods for many missing observations. Additionally, missing data as random or in chunks could also influence the comparisons of prediction accuracy depending on the dataset (Donders et al., 2006). Interpretations may also be influenced by the choice of error metric (e.g., RMSE) as different metrics have different objectives (Yozgatligil et al., 2013).

This paper describes the imputeTestbench package to simultaneously compare different imputation methods for univariate time series (Bokde and Beck, 2017). The goal of this package is to provide an evaluation toolset that addresses the above challenges for identifying an appropriate imputation method before more detailed analysis. This package provides several options for simulating missing observations with repeated sampling from a complete dataset. Missing values are imputed using any of several methods and then compared with a common error metric chosen by the user. Plotting functions are available to visualize the simulation methods for missing data, the predicted time series from each method, and overall evaluation of prediction accuracy between methods. Example applications are provided to demonstrate how imputeTestbench can be used to understand why different methods have different prediction accuracy given characteristics of the original data and characteristics of the missing data.

\section{Overview of imputeTestbench}

The theoretical foundation of imputeTestbench is shown in Figure 1. Comparing imputation methods begins by identifying the range of missing observations to remove and the number of repetitions for randomly removing the data. The removed data are imputed using one of several methods for each percentage of missing observations up to the maximum. Error metrics are used to identify the prediction accuracies of each imputation method for each repetition and interval of missing data.

Components of the workflow in Figure 1 are executed with the functions in imputeTestbench. The primary function is impute_errors() which is used to evaluate different imputation methods with missing data that are randomly generated from a complete dataset. The sample_dat() function is used to generate missing data within impute_errors() and includes a plotting option to demonstrate how the missing data are generated. The default error metrics for the imputed data are in the error_functions() function. The remaining two functions, plot_impute() and plot_errors(), are used to visualize imputation results and error summaries for the chosen methods. Dependencies include additional packages for data manipulation (dplyr, Wickham and Francois (2016); reshape2, Wickham (2007); tidyr, Wickham (2017)), graphing (ggplot2, Wickham (2009)), and imputation (forecast, Hyndman et al. (2017); imputeTS, Moritz and Bartz-Beielstein (2017); zoo, Zeileis and Grothendieck (2005)). 


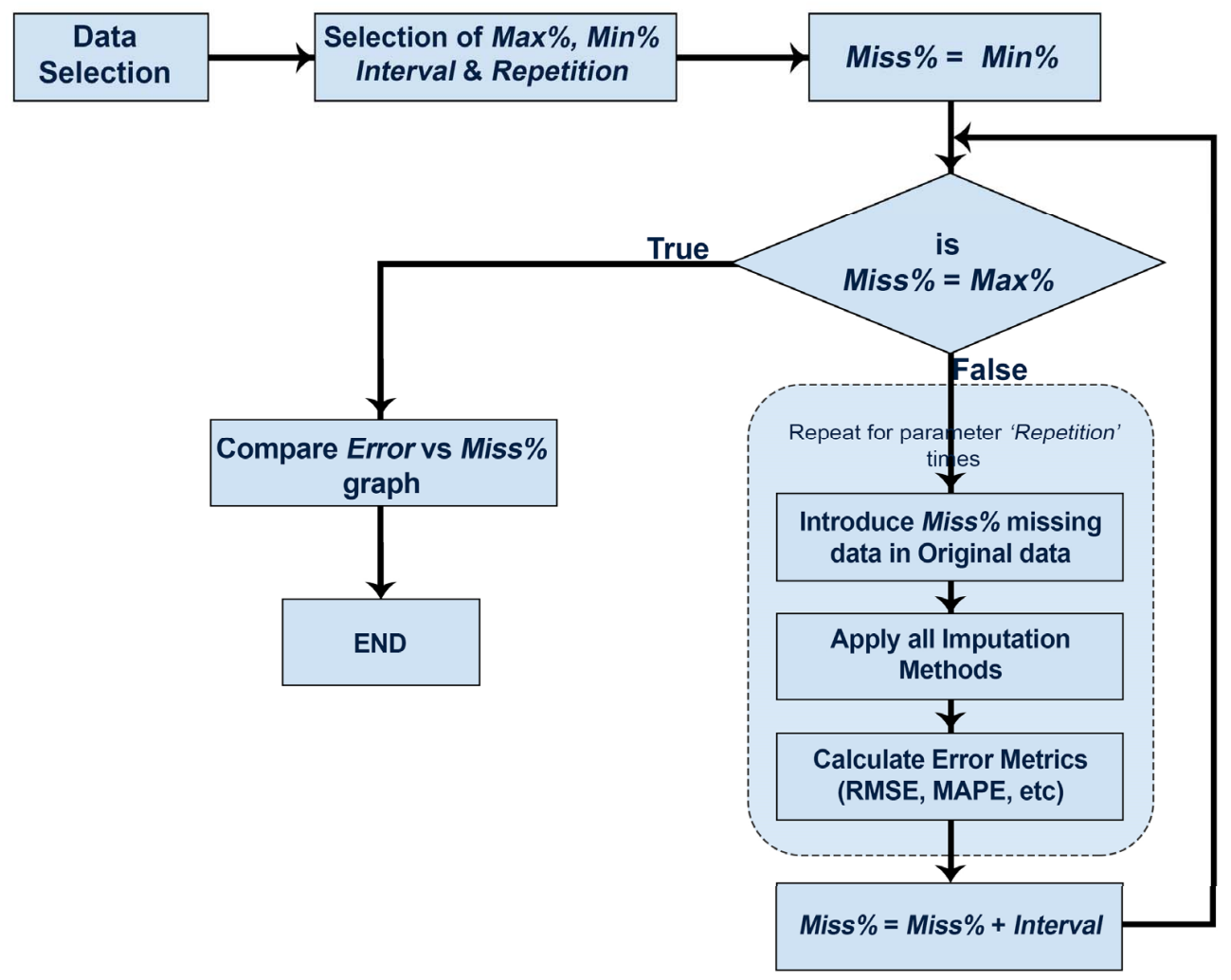

Figure 1: Workflow diagram for comparing imputation methods. Min $\%$ and Max $\%$ are minimum and maximum percent of missing values in the dataset. Details are described below.

\section{The impute_errors() function:}

The impute_errors() function evaluates the accuracy of different imputation methods based on changes in the amount and type of missing observations from the complete dataset. The default methods included in impute_errors() are three methods for linear interpolation (na.approx(), zoo; na. interp(), forecast; na. interpolation(), imputeTS), last-observation carried forward (na. locf(), zoo), and mean replacement (na.mean(), imputeTS). These methods are routinely applied in time series analysis, are easily understood compared to more complex approaches, and have relatively short computation times (Moritz and Bartz-Beielstein, 2017). Moreover, these methods represent a gradient from none to more complex dependence on the serial correlation of the time series - replacing missing data with overall means (na.mean()), replacing missing data with the last prior observation (na.locf()), and gap interpolation with linear methods (na.approx(), na.interp(), na. interpolation()). Note that the three linear methods vary considerably in the optional arguments that affect the imputation output. An expectation with the default methods included in imputeTestbench is varying imputation accuracy based on how each method relies on characteristics of a dataset to predict missing observations. Although we acknowledge that the effectiveness of a chosen method depends on the data, the default techniques represent a broad range that is sufficient for most applications. As noted below, additional methods can be added as needed.

The impute_errors() function has the following arguments:

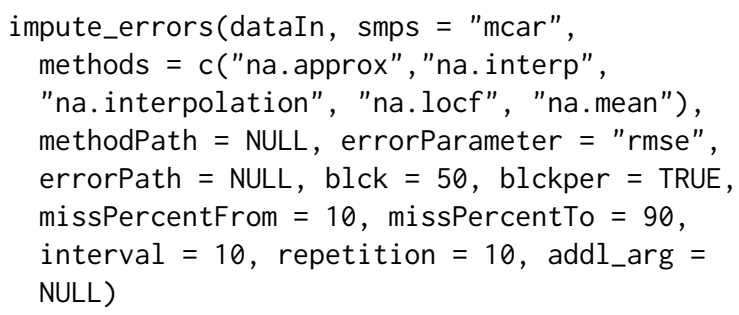


dataIn:

A ts (stats) or numeric object that will be evaluated. The input object is a complete dataset to evaluate by simulating missing data for performance evaluation and comparison of imputation methods. The examples in the documentation use the nottem time series object of average air temperatures recorded at Nottingham Castle from 1920 to 1930 (datasets package).

smps:

The desired type of sampling method for removing values from the complete time series provided by dataIn. Options are smps = 'mcar' for missing completely at random (MCAR, default) and smps = ' mar' for missing at random (MAR). Both methods provide different approaches to generating missing data in time series. In general, MCAR removes individual observations where the likelihood of a single observation being removed does not depend on whether observations closer in time have also been removed. By contrast, MAR removes observations in continuous blocks such that the likelihood of an observation being removed depends on whether observations closer in time have also been removed. The methods are described in detail in the section Sampling methods for missing observations.

methods:

Methods that are used to impute the missing values generated by smps: replace with means (na. mean()), last-observation carried forward $(\operatorname{na} . \operatorname{loc} f())$, and three methods of linear interpolation (na.approx(), na.interp(), na. interpolation()). All five default methods are used unless the argument is changed by the user. For example, methods = 'na. approx' will use only na. approx() with impute_errors(). Methods not included with the default options can be added by including the name of the function in methods and providing the path to the script in methodPath. Additional arguments passed to each method can be included in addl_arg described below.

methodPath:

A character string for the path of the user-supplied script that includes one to many functions passed to methods. The path can be absolute or relative within the current working directory for the R session. The impute_errors() function sources the file indicated by methodPath to add the user-supplied function to the global environment.

\section{errorParameter:}

The error metric used to compare the true, observed values from dataIn with the imputed values. Metrics included with imputeTestbench are root-mean squared error (RMSE), mean absolute percent error $(M A P E)$ and mean absolute error $(M A E)$. The metric can be changed using errorParameter = 'rmse' (default), 'mape', or 'mae'. Formulas for the error metrics are as follows:

$$
\begin{gathered}
R M S E=\sqrt{\frac{\sum_{i=1}^{n}\left(x_{i}-\widehat{x}_{i}\right)^{2}}{n}} \\
M A P E=100 \cdot \frac{\sum_{i=1}^{n}\left|\left(x_{i}-\widehat{x}_{i}\right) / x_{i}\right|}{n} \\
M A E=\frac{\sum_{i=1}^{n}\left|x_{i}-\widehat{x}_{i}\right|}{n}
\end{gathered}
$$

where $n$ is the total number of missing observations, $x$ are the actual observations, and $\widehat{x}$ are the imputed observations. Additional error measures can be provided as user-supplied functions.

errorPath:

A character string for the path of the user-supplied script that includes one to many error methods passed to errorParameter. 
blck:

The block size for missing data if the sampling method is at random, smps = 'mar'. The block size can be specified as a percentage of the total amount of missing observations to remove or as a number of time steps in the input dataset. For example, if blck $=50$ and blkper $=$ TRUE (indicating blck is a percentage), each block has a size that is $50 \%$ of the total amount of observations to remove. A time series with 100 observations will have two missing chunks of 20 observations each if a total of $40 \%$ of the observations are removed and each chunk is $50 \%$ of the total. The total amount of observations to remove depends on values inherited from missPercentFrom, missPercentTo, and interval.

\section{blckper:}

A logical value indicating if the value for blck is a percentage (blckper = TRUE) of the total number of observations to remove or a sequential number of time steps (blckper $=$ FALSE) to remove for each block. This argument only applies if smps = 'mar'.

\section{missPercentFrom, missPercentTo:}

The minimum and maximum percentages of missing values, respectively, that are introduced in dataIn. Appropriate values for these arguments are 10 to 90, indicating a range from few missing observations to almost completely absent observations.

\section{interval:}

The interval of missing data from missPercentFrom to missPercentTo. The default value is $10 \%$ such that missing percentages in dataIn are evaluated from $10 \%$ to $90 \%$ at an interval of $10 \%$, i.e., $10 \%, 20 \%$, $30 \%, \ldots, 90 \%$. Combined, these arguments are identical to seq $($ from $=10$, to $=90$, by $=10)$.

\section{repetition:}

The number of repetitions at each interval. Missing values are placed randomly in the original data such that multiple repetitions must be evaluated for a robust comparison of the imputation methods.

Considering the default values for the above arguments, the impute_errors() function returns an "errprof" object as the error profile for the imputation methods:

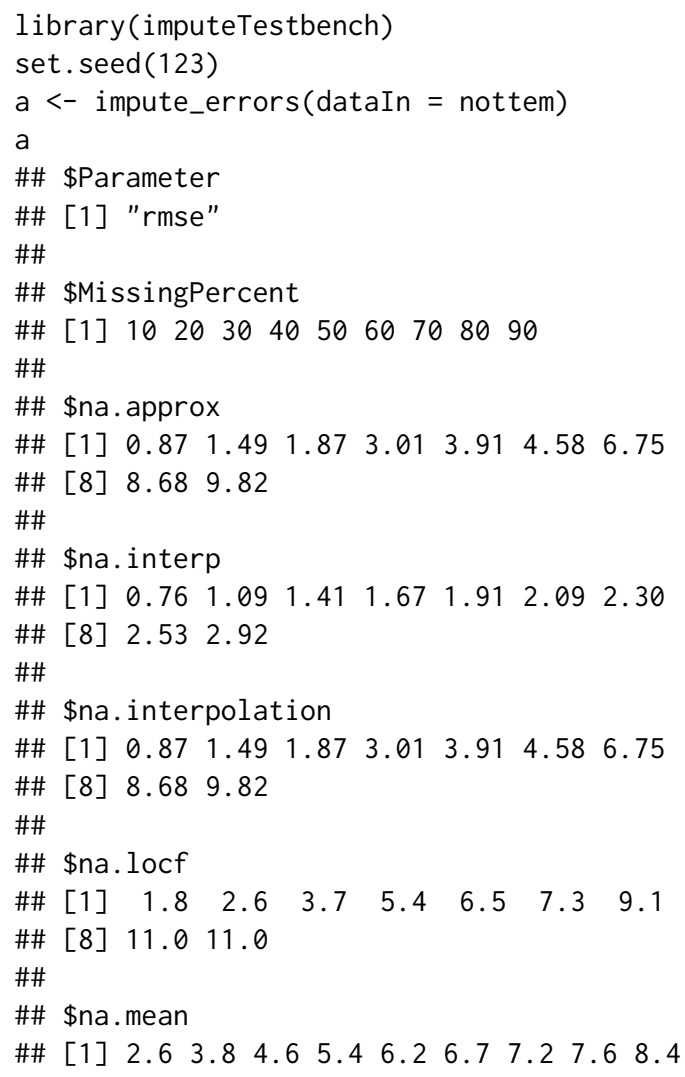


The "errprof" object is a list with seven elements. The first element, Parameter, is a character string of the error metric used for comparing imputation methods. The second element, MissingPercent, is a numeric vector of the missing percentages that were evaluated in the input dataset. The remaining five elements show the average error for each imputation method at each interval of missing data in MissingPercent. The averages at each interval are based on the repetitions specified in the initial call to impute_errors () , where the default is repetition $=10$. Although the print method for the "errprof" object returns a list, the object stores the unique error estimates for every imputation method, repetition, and missing data interval. These values are used to estimate the averages in the printed output and to plot the distribution of errors with plot_errors() shown below. All error values can be accessed from the errall attribute, i.e., attr (a, 'errall').

\section{Viewing results from impute_errors()}

The plot_errors() function can be used to view summaries of the imputation errors for each method. This function uses the "errprof" object as input and returns a graph of error values to compare results from the methods across the range of missing data. Three plot types are provided by plot_errors() and are specified with the plotType argument using one of three values: "boxplot", "bar", or "line". The default value is plotType = 'boxplot' that graphs the distribution of error values for each method and missing data interval using boxplot summaries (i.e., 25th, 50th, and 75th percentile shown by the box, whiskers as 1.5 times interquartile range, and outliers beyond). The boxplots are created using all error values stored in the 'errall' attribute of the "errprof" object (Figure 2).

plot_errors(a)

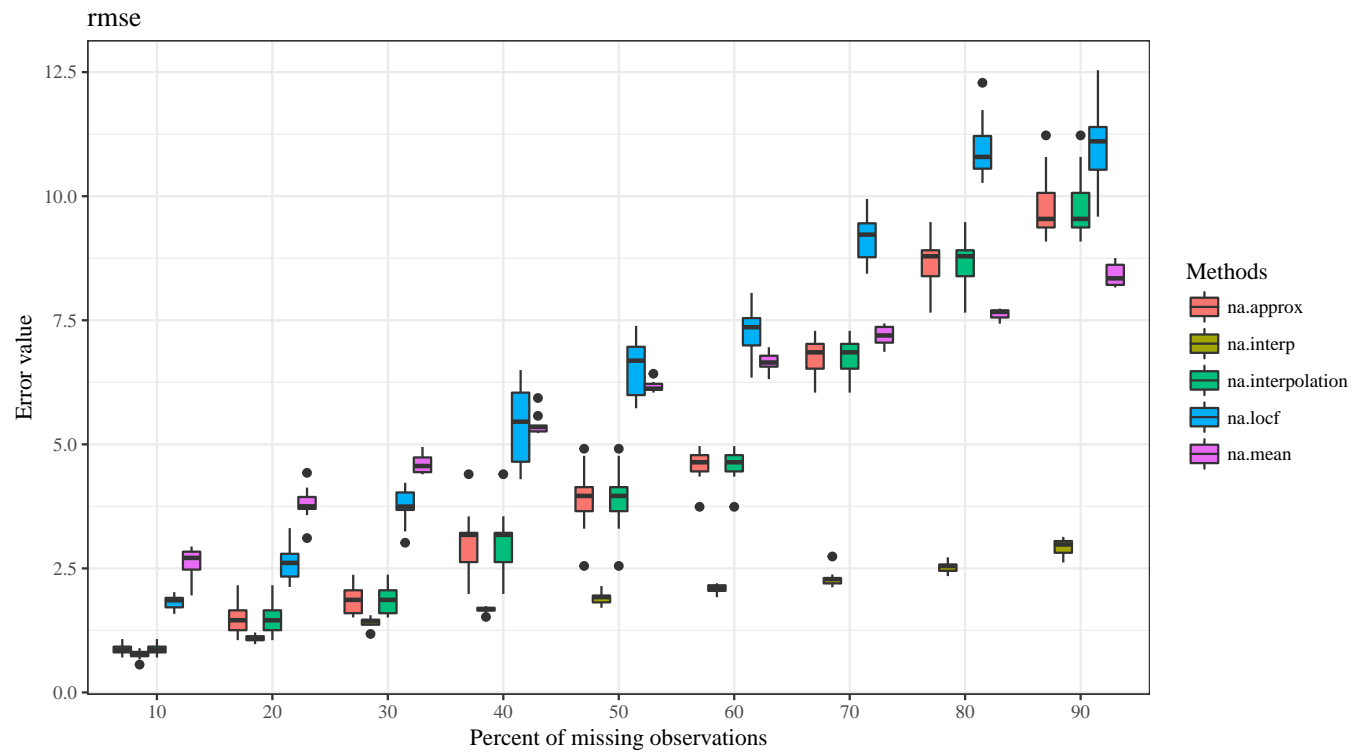

Figure 2: Distribution of error values from the "errprof" object for each imputation method and interval of missing observations. The plot is created with plot_errors() using the boxplot option.

The bar and line options for plotType show the average error values for each repetition. Similar information is shown as the boxplot option, although the range of error values for each imputation method is not shown ('line' option shown in Figure 3).

plot_errors $(a, \operatorname{plotType}=$ 'line' $)$

\section{Sampling methods for missing observations}

The impute_errors() function uses sample_dat() to remove observations for imputation from the input dataset. Observations are removed using one of two methods relevant for univariate time series: MCAR and MAR. The MCAR sampling scheme assumes all observations have equal probability of being selected for removal and is appropriate for understanding imputation accuracy with univariate time series that are not serially correlated (Rubin, 1976). Conversely, the MAR sampling scheme selects observations in blocks such that the probability of selection for a single observation depends on 


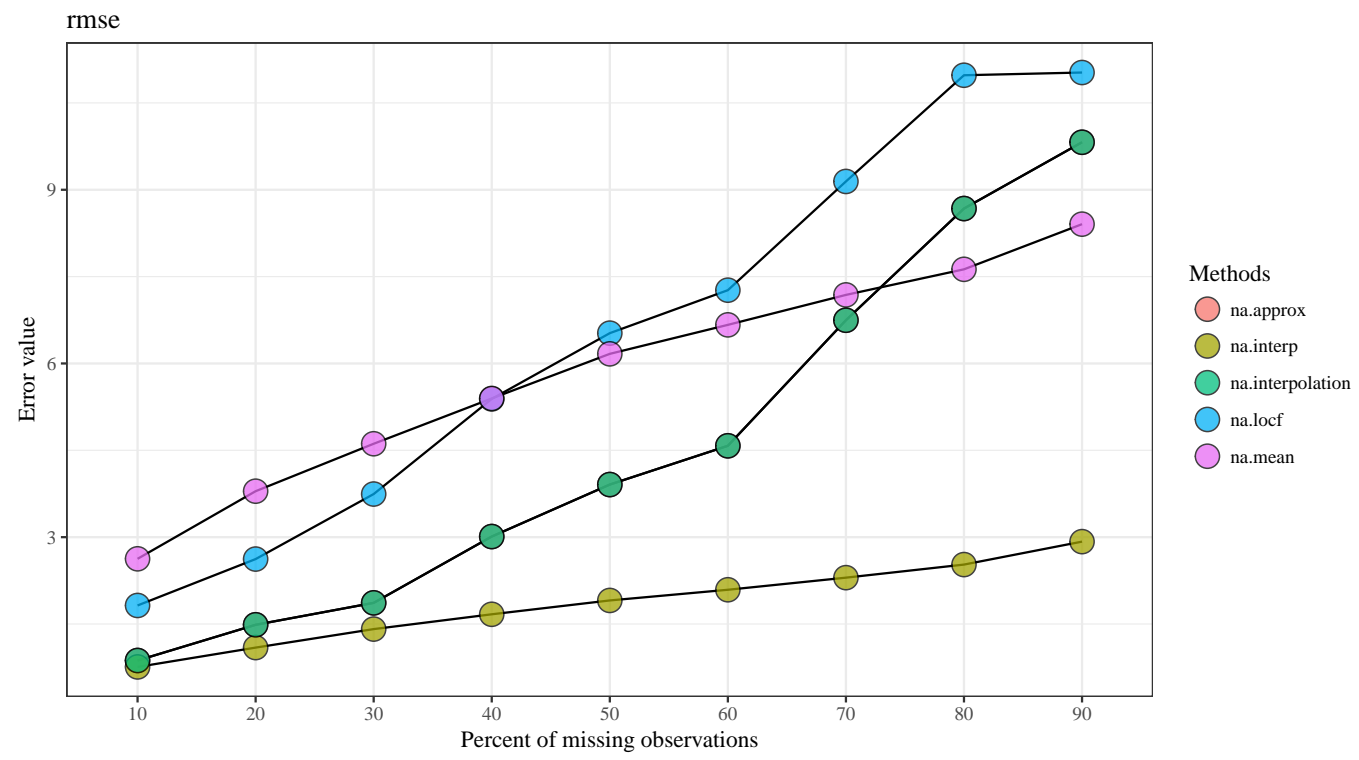

Figure 3: Average error values for each imputation method and interval of missing observations. The line option is used for plot_errors().

whether an observation closer in time was also selected (Schafer and Graham, 2002). The MAR scheme is appropriate for time series with serial correlation. The sample_dat() function has the following syntax:

sample_dat(datin, smps $=$ "mcar", repetition $=10, \mathrm{~b}=50$, blck $=50$, blckper $=$ TRUE, plot $=$ FALSE)

datin:

Input "ts" object or numeric vector, inherited from dataIn from impute_errors().

smps, repetition, blck, blckper:

Arguments that are inherited as is from impute_errors() indicating the sampling type (smps), number of repetitions for each missing data type (repetition), block size (blck), and block type as percentage or count (blckper).

b:

Numeric indicating the total amount of missing data as a percentage to remove from the complete time series. The arguments missPercentFrom, missPercentTo, and interval from impute_errors() define $b$ for each simulation of missing observations with sample_dat (). For example, missing data will be simulated with sample_dat() for percentages of the total sample size as $b=10,20, \ldots, 90$ if missPercentFrom $=10$, missPercentTo $=90$, and interval $=10$ for impute_errors () .

plot:

Logical indicating if a plot is returned that shows one repetition of the sampling scheme defined by the arguments (Figure 4).

The MCAR sampling scheme is used if smps = 'mcar', where the only relevant arguments are missPercentFrom, missPercentTo, and interval from impute_errors() for the missing data. The amount of data to remove for each interval is passed to the $b$ argument in sample_dat (). Alternatively, the MAR sampling scheme requires additional arguments to control the block size for removing data in continuous chunks, in addition to the total amount of data to remove defined by $b$. The block size argument, blck, can be given as a percentage or as number of observations in sequence. The type of block size passed to blck is controlled by blckper, where blckper = TRUE indicates a percentage and FALSE indicates a count for blck. For example, if the total sample size of the dataset is 1000, b 
$=50$, blck $=20$, and blckper $=$ TRUE means half the dataset is removed $(b=50,500$ observations $)$ and each block will have 100 observations (20\% of 500). For both percentages and counts, the blocks are automatically selected until the total amount of missing data is equal to that specified by b. Final blocks may be truncated to make the total amount of missing observations equal to $b$. The starting location of each block is selected at random and overlapping blocks are not uniquely counted for the required sample size given by b (i.e., blocks larger than that specified by blck may occur of two separate blocks overlap).

The sample_dat() function is typically not used independently of impute_errors(), although an optional plotting argument is provided to visualize different sampling schemes for removing data. Figure 4 shows examples of sampling with MCAR and MAR.

\section{The plot_impute() function}

The third plotting function available in imputeTestbench is plot_impute(). This function returns a plot of the imputed values for each imputation method in impute_errors () for one repetition of sampling for missing data. The plot shows the results in individual panels for each method with the points colored as retained or imputed (i.e., original data not removed and imputed data that were removed). An optional argument, showmiss, can be used to plot the original values as open circles that were removed from the data. The plot_impute() function shows results for only one simulation and missing data type (e.g., smps $=$ 'mcar' and $b=50$ ). Although the plot from plot_errors () is a more accurate representation of overall performance of each method, plot_impute ( $)$ is useful to better understand how the methods predict values for a sample dataset (Figure 5).

plot_impute $($ dataIn $=$ nottem, showmiss $=T)$

\section{Including additional arguments}

Additional arguments for the imputation functions used by impute_errors() can easily be modified. This is useful for changing the default arguments, particularly for the three linear interpolation methods (na.approach(), na.interp(), na.interpolation()). Although these methods are very similar in the default configuration, the amount of flexibility varies considerably. For example, na. interpolation() provides spline interpolation as an alternative that is not provided by the other functions. Using each method without modifying the additional arguments is not suggested given that no information is gained by comparing the linear interpolation methods with the default setup.

Accordingly, additional arguments can be passed to any of the imputation methods using the addl_arg argument. These additional arguments are passed as a list of lists, where the list contains one to many elements that are named by the methods in methods. The elements of the list are lists with arguments that are specific to each imputation method. For example, the default imputation function na. mean() has an additional option argument that specifies the algorithm for missing values, where possible values are "mean", "median", and "mode". This argument can be changed from the default option = "mean" with addl_arg in impute_errors(), as shown below. Arguments to user-supplied imputation functions (below) can be changed similarly.

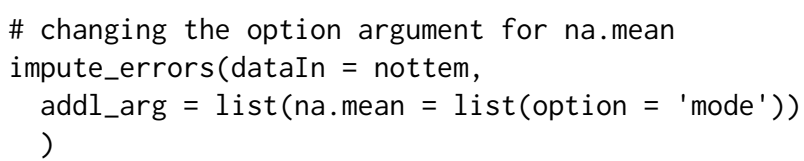

\section{Adding imputation methods and error metrics}

Additional imputation methods saved as an R script can be added to impute_errors(). Attention should be given to the format of the user-supplied function shown below. The time series data (as numeric or "ts" object) with missing values is required as input and the return value is the input dataset with imputed values, where the input and output objects have the same length. The impute_errors () function will not process the data correctly if the format is incorrect. The file path for the R script is supplied as an input string to the methodPath argument and the function name is added to the methods argument for the impute_errors() function.

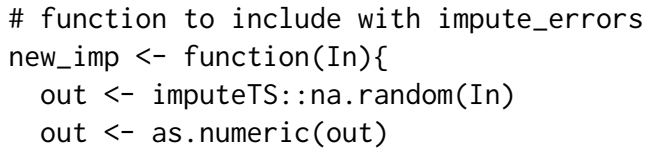


$\mathrm{b}=10$, smps $=$ "mcar"

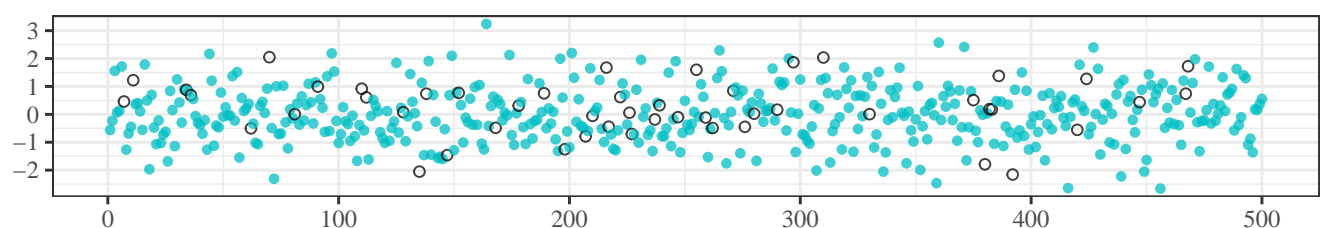

$\mathrm{b}=50$, smps $=$ "mcar"

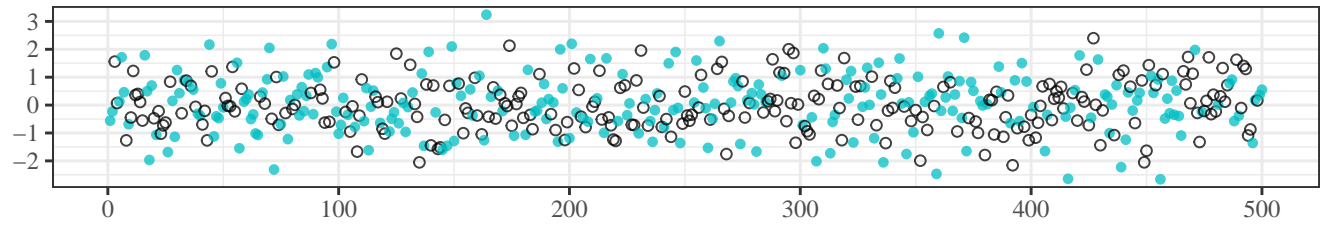

$\mathrm{b}=10$, smps $=$ "mar", blck $=10$, blckper $=$ TRUE

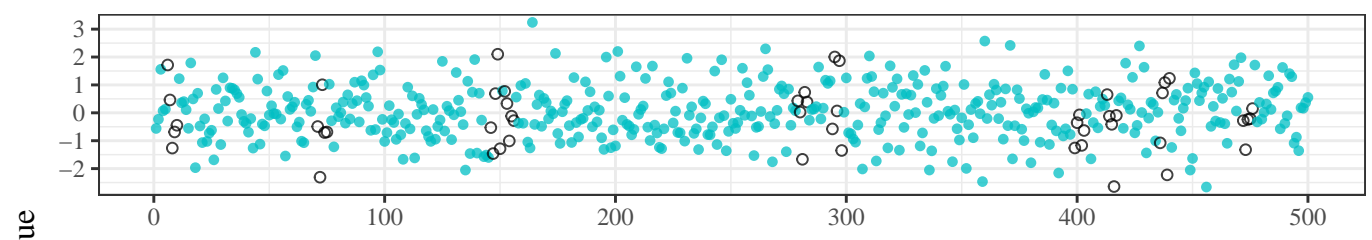

蛋 $\mathrm{b}=50$, smps $=$ "mar", blck $=50$, blckper $=$ TRUE

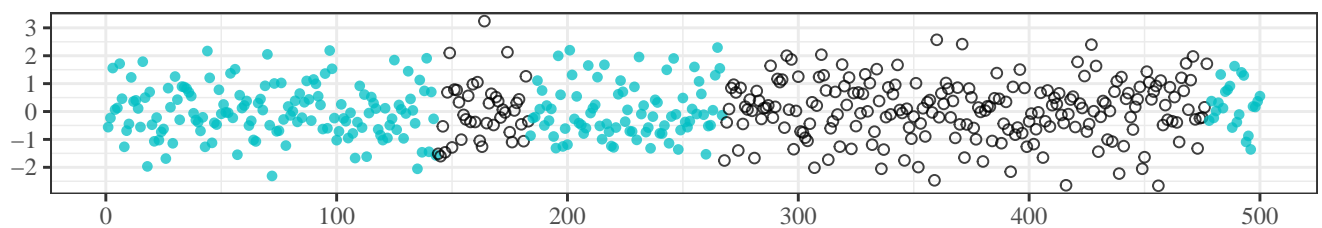

$\mathrm{b}=10$, smps $=$ "mar", blck $=10$, blckper $=$ FALSE

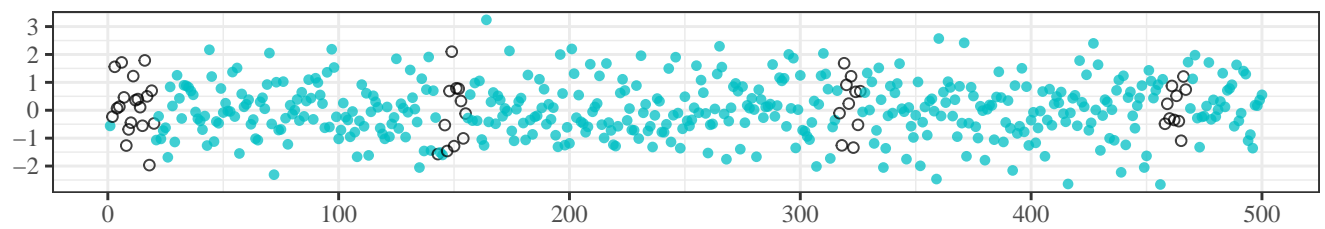

$\mathrm{b}=50, \mathrm{smps}=$ "mar", blck $=50$, blckper $=$ FALSE

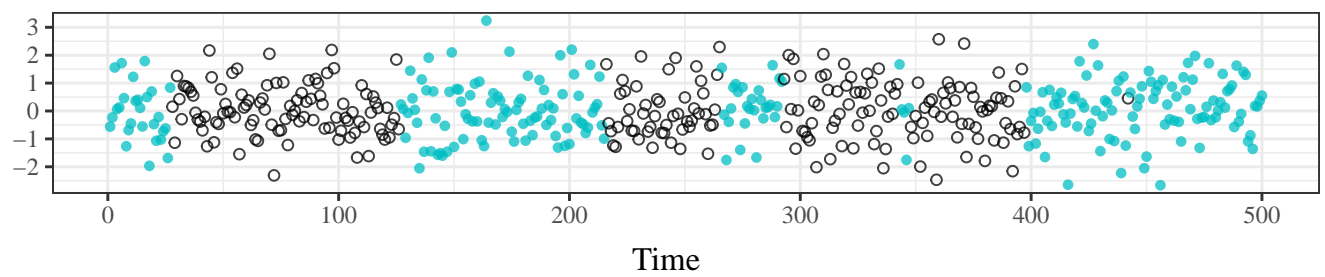

Figure 4: Examples of sampling schemes for missing data provided by sample_dat(), plotted using the argument plot $=\mathrm{T}$. Values to be removed and imputed are shown as open circles and the data to be kept are in blue. From top to bottom, sampling is MCAR with 10\% missing, MCAR with 50\% missing, MAR with $10 \%$ missing and block size $10 \%$ of total missing, MAR with $50 \%$ missing and block size $50 \%$ of total missing, MAR with $10 \%$ missing and block size of ten observations, and MAR with $50 \%$ missing and block size of fifty observations.

As described above, the error metrics included with imputeTestbench are RMSE, MAE, and $M A P E$. These metrics provide different information and contrasting approaches to evaluate imputation methods. For example, RMSE is a commonly used metric that maintains the scale of observations in the input data. The $M A E$ metric is similar but more interpretable as the differences are in proportion to the absolute values of the errors, which differs from RMSE that uses the sum of squared deviations. The MAPE metric is a simple extension of $M A E$ that scales the output by the range of observations in 
- Imputed $\odot$ Retained $\quad$ Removed

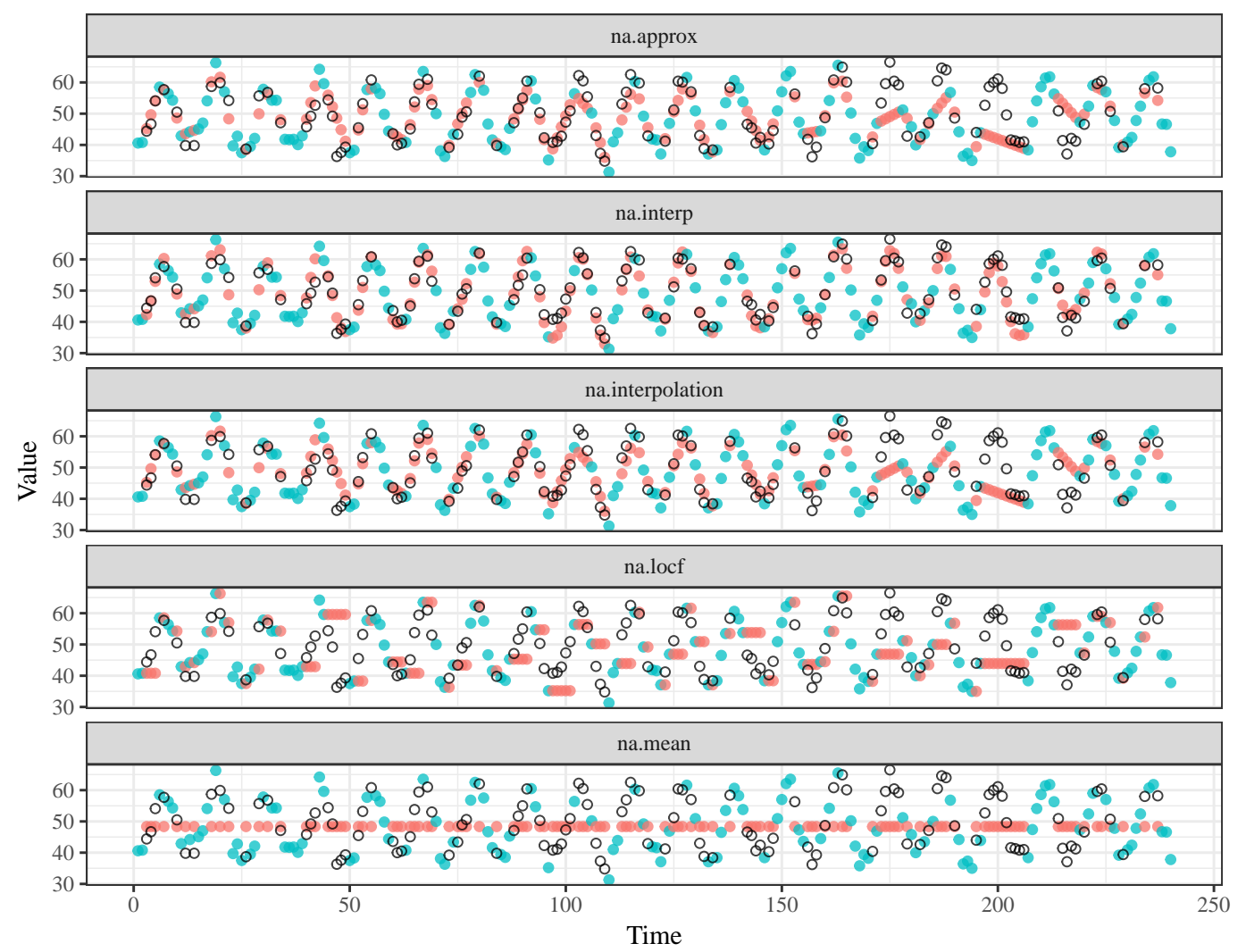

Figure 5: Output from the plot_impute() function that shows the data that were retained (blue), removed (open circles), and imputed (red).

the input data and is useful for comparing datasets that differ in scale. Users should choose a metric based on the information provided by each. For example, imputation comparisons with different datasets should use the MAPE metric that standardizes the scale of assessment.

Each of the metrics are called by the error_functions() function internally within impute_errors(). Additional error metrics are added using an approach similar to that used for adding imputation methods. The following example shows use of the percent change in variance (PCV, Tak et al. (2016)) as an alternative error metric:

$$
P C V=\frac{\operatorname{var}(\bar{V})-\operatorname{var}(V)}{\operatorname{var}(V)}
$$

The user-supplied error function must include two arguments as input, the first being a vector of observed values and the second being a vector of imputed missing values equal in length to the first. The function must also return a single value as a summary of the errors or differences. As before, the new error function should be saved as an $\mathrm{R}$ script. The file path is added to the errorPath argument and the error function name is added as a character string to the errorParameter argument for impute_errors().

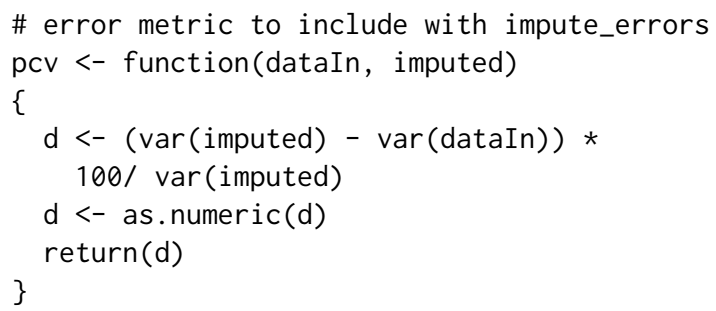




\section{Demonstration of imputeTestbench}

This example demonstrates how imputeTestbench can be used to compare imputation methods, and more importantly, how it can be used to better understand the effects of time series characteristics on prediction accuracies. The objective of the comparison is to relate prediction accuracies of each method to the time series characteristics of each dataset, including a description of how the amount and type of missing data influence the results. Three univariate datasets with different characteristics are evaluated (Figure 6). The first dataset, $\mathrm{nrm}$, is a sample of 100 random observations from a standard normal distribution to simulate a dataset with no temporal correlation. The second dataset, austres, is a "ts" object of Australian population in thousands, measured quarterly from 1971 to 1994 (Brockwell and Davis, 1996). This dataset includes a simple correlation structure with minimal random noise. The final dataset is nottem as described above. This dataset is characterized by a cyclical or repeating seasonal component. Lagged correlations show the differences in the temporal dependencies between the datasets (Figure 6).
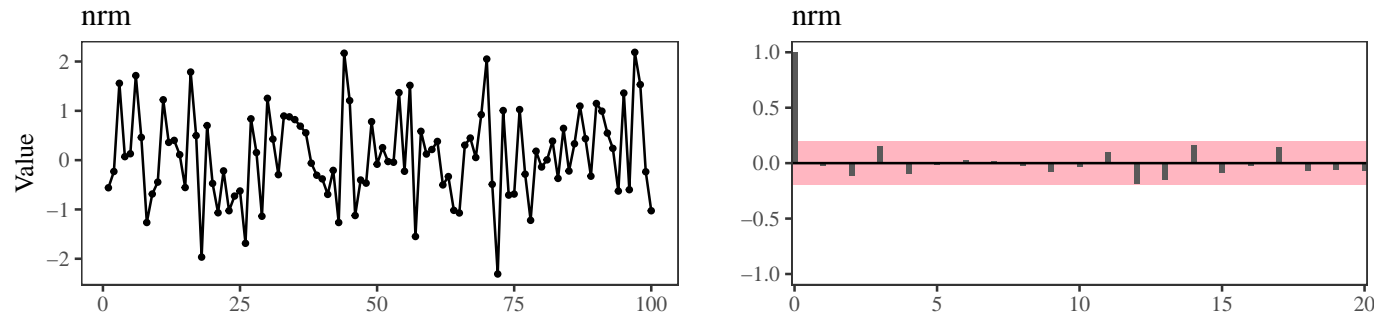

austres

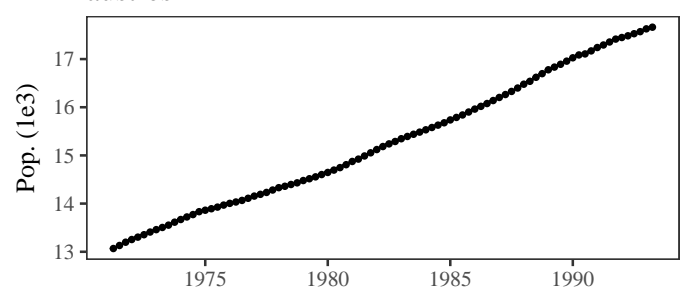

austres
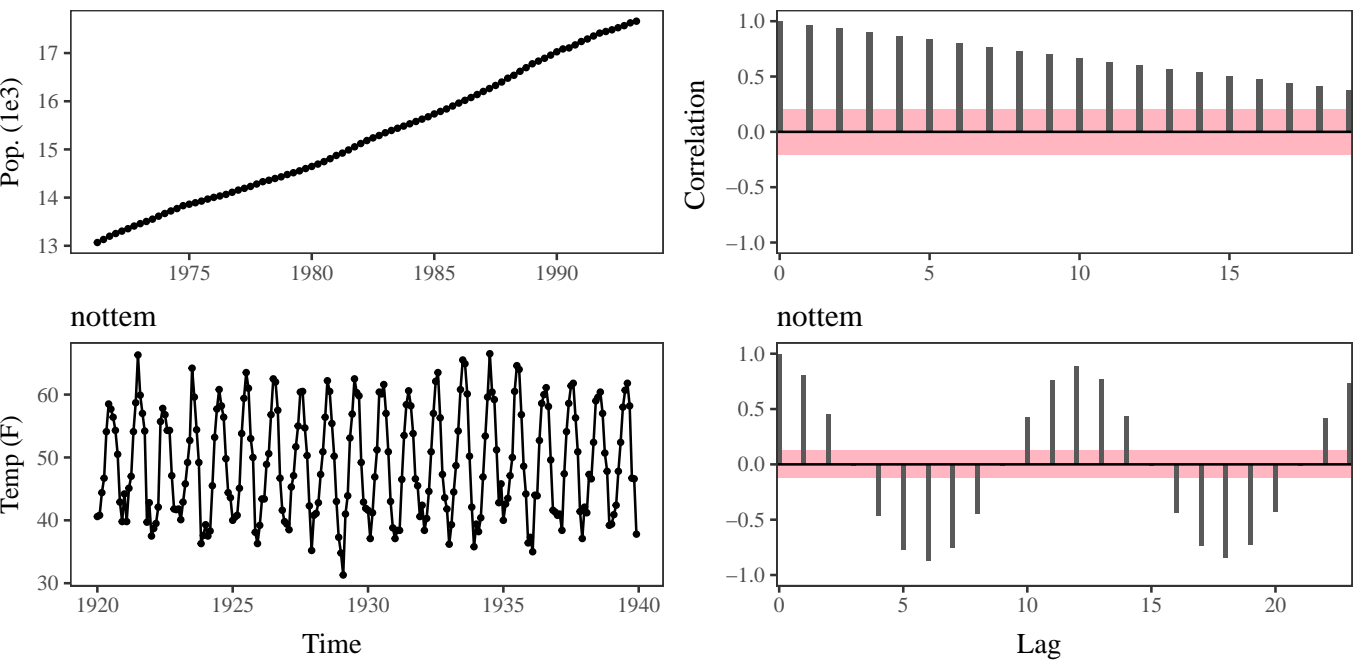

Figure 6: Three datasets used to demonstrate application of imputeTestbench. The top dataset, nrm, was created with random samples from a normal distribution. The bottom two datasets are austres and nottem from the datasets package. Each dataset has different temporal correlation structures shown at different lags (right column). The light red shading indicates a threshold beyond which correlations are significant $(\alpha=0.05)$.

Each dataset was evaluated by simulating missing observations from $10 \%$ to $90 \%$ of the complete data using $\operatorname{MCAR}($ smps = 'mcar') and MAR (smps = 'mar') sampling. The size of each chunk (blck argument) for MAR sampling was evaluated at $20 \%$ and $100 \%$ of the total percentage of missing observations to evaluate an effect of chunk size (small chunks to one large chunk) on imputation accuracy. As such, the analysis evaluated imputation accuracy between the default methods as affected by dataset type ( $\mathrm{nrm}$ - no correlation structure, austres - simple correlation, not tem - seasonal correlation) and characteristics of the missing observations (MAR, MCAR, varying amounts of missing data and chunk sizes). All comparisons used the MAPE metric to evaluate imputation errors given scale differences between the datasets. The following code demonstrates the analysis setup.

\# load packages, set seed

library (tidyverse)

library (imputeTestbench)

set. seed(123) 

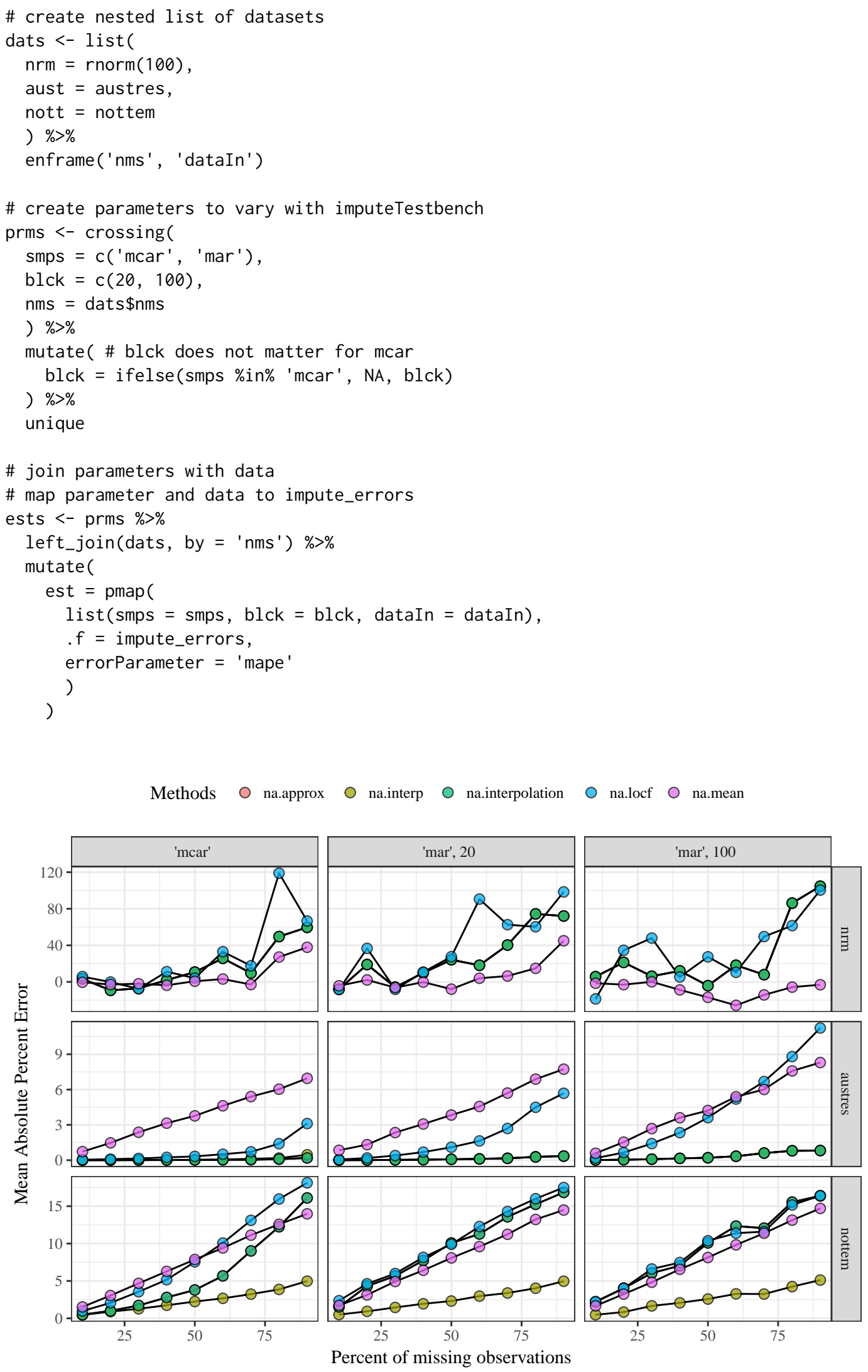

Figure 7: Comparisons of prediction errors for three datasets with different temporal correlation structures (see Figure 6). Missing observations were simulated from the complete datasets as MCAR and MAR with missing chunks of different sizes from $20 \%$ and $100 \%$ of the total. The total percentage of missing observations from the complete datasets varied from $10 \%$ to $90 \%$.

The prediction errors varied considerably between the datasets, imputation method, and type 
of simulation for missing observations (Figure 7). As expected, the type of simulation (MCAR and MAR with different chunk sizes) did not have a noticeable influence on prediction accuracy for the normally distributed dataset with no correlation structure $(\mathrm{nrm})$. This dataset is described by only two parameters (mean and standard deviation) and the observations are completely independent such that imputation accuracy was similar for both MCAR and MAR sampling, although accuracy decreased with the addition of missing observations which is not unexpected. The na.mean () function generally outperformed the other imputation methods that incorporate some level of information about the relationship between observations. The $\mathrm{nrm}$ dataset has observations that are not correlated and the use of imputation methods that leverage temporal dependence in the input dataset is expected to induce bias in the imputation, as shown by larger errors.

By comparison, the prediction errors for the austres and not tem datasets differed in both the type of simulation for missing observations and the imputation method. For austres, linear interpolations consistently produced imputations with the least error and each of the three linear interpolation methods (na. approx(), na. interp(), na. interpolation()) performed equally well regardless of simulation type for missing data. This result is not surprising given that austres can be described as a monotonic linear increase with minimal variation. That is, imputing a straight line between observations reproduces the original dataset with high accuracy. However, relative prediction accuracies between methods were affected by the type of simulation for removing observations. Imputations with na. $\operatorname{loc} f()$ method had increasing error with increasing size of the missing chunk and errors exceeded na. mean() for large chunk sizes and high percentages of missing data (i.e., $>60 \%$ missing as one large chunk). These results can be explained by viewing sample imputations with plot_impute() (Figure (8). As in Figure 7, the na. mean() function performs poorly for most scenarios because it does not capture the linear increase through time. However, the na. locf() and na. approx () functions perform equally well for small percentages of missing data but error values diverge for larger percentages. Errors for na. $\operatorname{locf}()$ exceed those for na. mean() when large chunks are removed given that the latter produces less biased estimates, although the method performs poorly overall.

\section{- Imputed $\odot$ Retained $\bigcirc$ Removed}

Removed as 'mcar'

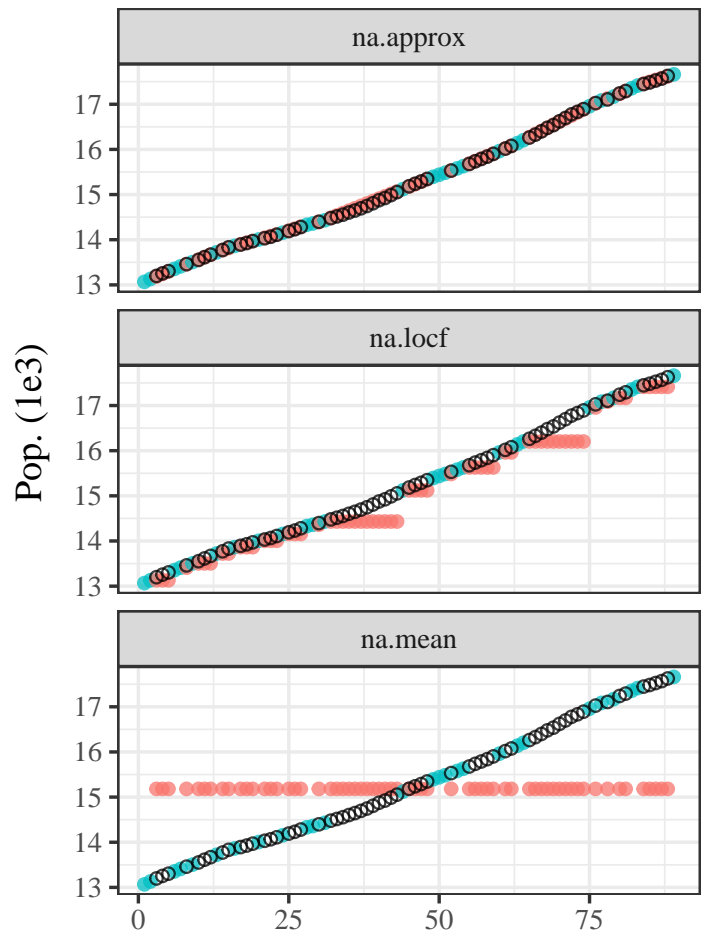

Removed as 'mar', one chunk

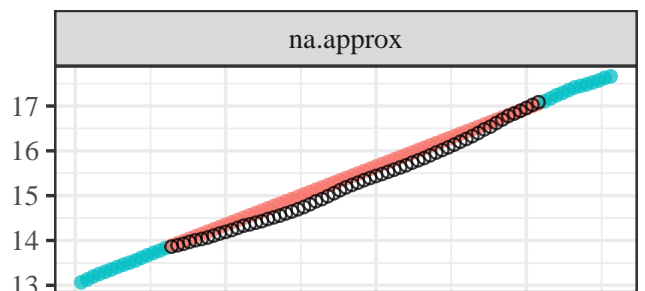

na.locf

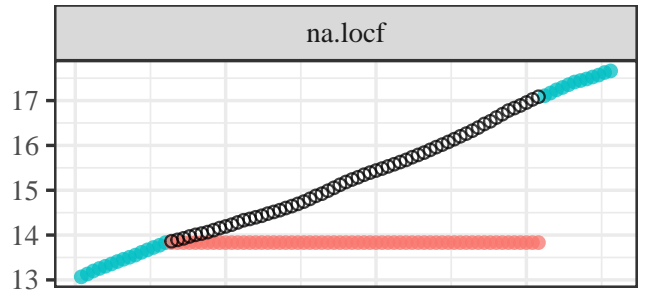

na.mean

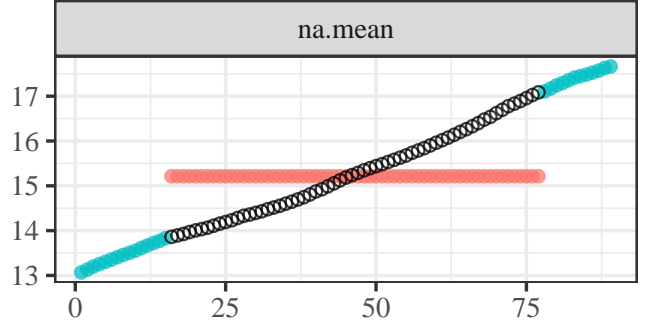

Time

Figure 8: An example from plot_impute() of imputed values for the austres dataset using na.approx, na.locf, and na.mean. Seventy percent of observations were removed for each dataset. The left column shows the observations removed using MCAR and the right shows the observations removed using MAR.

Finally, imputations of the nottem dataset had similar prediction errors independent of how 
the data were removed, although the imputation methods varied considerably. Specifically, the na.interp() function consistently had lower prediction errors for all percentages of missing observations. Interestingly, the na.approx() and na. interpolation() functions did not have similar performance as na.interp(), although all three methods are similarly documented as linear interpolations. Examples in Figure 5 demonstrate differences in the imputations between the three methods. Both na.approx() and na. interpolation() do not describe the seasonal variation in the data that is well-described by na.interp(). As noted in the documentation, na.interp() optionally performs periodic decomposition for seasonal time series based on characteristics of the input data (Hyndman et al., 2017). This highlights the need to carefully understand the assumptions of each method and that the default behavior between ostensibly similar approaches could vary. Users should be familiar with the help documentation and are advised to make use of the addl_args argument in impute_errors() to modify the default behavior of the chosen imputation functions. The imputeTestbench package provides sufficient flexibility to accommodate differences among methods for each approach evaluated with impute_errors().

\section{Summary}

The applied example demonstrated the value of imputeTestbench for informing the use of imputation methods as a necessary step towards more formal time series analysis. For all examples, a complete dataset was used to demonstrate how characteristics of the temporal correlation structure can influence the prediction accuracy. The default imputation methods in the package have different approaches to imputing missing values that vary in the amount of dependence on the correlation structure of input data. As expected, the accuracy of each imputation method varied depending on the dataset and a general conclusion is that users should carefully evaluate the correlation structure and periodic components of actual data as an approach to choosing an imputation method. The imputeTestbench package greatly facilitates this preliminary step by simultaneously comparing different methods and considering the type and amount of missing observations. In practice, observational data that contain missing values cannot be used directly with the package because the intent is to evaluate hypothetical scenarios of missing observations with complete data. As such, the sample dataset used with imputeTestBench should have characteristics similar to the dataset for which imputation is needed. Our applied example evaluated three time series data with different characteristics and additional comparisons of datasets with more complex temporal dependencies could be helpful towards informing practical applications.

We also demonstrated how the package can be modified to include additional imputation methods or comparison metrics. By default, the package provides a core set of existing imputation methods (na.approx(), na.interp(), na.interpolation(), na. $\operatorname{locf}()$, and na.mean()), which are simultaneously compared using RMSE, MAE, or MAPE error metrics. These simple methods will likely be sufficient for most users, although we recognize that the comparison of other methods and error metrics will be required in more advanced cases. As such, the package allows users to include additional imputation methods for comparison, which could be particularly useful given the capability of $\mathrm{R}$ to interface with other programming languages (e.g, Rcpp for compiled languages, Eddelbuettel and François (2011); matlabr for MatLab, Muschelli (2016)). As such, the simple architecture of imputeTestbench to add or remove multiple methods and error metrics makes it a robust and useful tool to evaluate existing and proposed imputation techniques for univariate time series.

\section{Acknowledgments}

We thank the R user community for providing feedback that improved earlier versions of the software. The views expressed in this paper are those of the authors and do not necessarily reflect the views or policies of the U.S. Environmental Protection Agency.

\section{Bibliography}

N. Bokde and M. W. Beck. imputeTestbench: Test Bench for the Comparison of Imputation Methods, 2017. URL https://cran. $r$-project. org/package=imputeTestbench. R package version 3.0.1. [p219]

G. E. P. Box, G. M. Jenkins, G. C. Reinsel, and G. M. Ljung. Time Series Analysis: Forecasting and Control. John Wiley \& Sons, Hoboken, New Jersey, 5th edition, 2015. ISBN 978-1-118-67502-1. [p218]

P. J. Brockwell and R. A. Davis. Introduction to Time Series and Forecasting. Springer-Verlag, 1996. ISBN 978-1-4757-2526-1. [p228] 
A. R. T. Donders, G. J. M. G. van der Heijden, T. Stijnen, and K. G. M. Moons. Review: A gentle introduction to imputation of missing values. Journal of Clinical Epidemiology, 59:1087-1091, 2006. URL https://doi.org/10.1016/j.jclinepi.2006.01.014. [p218,219]

D. Eddelbuettel and R. François. Rcpp: Seamless R and C++ integration. Journal of Statistical Software, 40(8):1-18, 2011. URL https://doi.org/10.18637/jss.v040.i08. [p231]

V. Gomez and A. Maravall. Estimation, prediction, and interpolation for nonstationary series with the Kalman filter. Journal of the American Statistical Association, 89(426):611-624, 1994. URL https: //doi.org/10.1080/01621459.1994.10476786. [p218]

P. G. Gould, A. B. Koehler, J. K. Ord, R. D. Snyder, R. J. Hyndman, and F. Vahid-Araghi. Forecasting time series with multiple seasonal patterns. European Journal of Operational Research, 191(1):207-222, 2008. URL https://doi.org/10.1016/j.ejor.2007.08.024. [p218]

J. Honaker and G. King. What to do about missing values in time-series cross-section data. American Journal of Political Science, 54(2):561-581, 2010. URL https://doi .org/10.1111/j .1540-5907.2010. 00447.x. [p218]

R. J. Hyndman, M. O'Hara-Wild, C. Bergmeir, S. Razbash, and E. Wang. Forecast: Forecasting Functions for Time Series and Linear Models, 2017. URL http://gi thub.com/robjhyndman/forecast. R package version 8.1. [p219, 231]

R. Jörnsten, M. Ouyang, and H.-Y. Wang. A meta-data based method for DNA microarray imputation. BMC Bioinformatics, 8(1):109, 2007. URL https://doi .org/10.1186/1471-2105-8-109. [p219]

H. Li, C. Zhao, F. Shao, G.-Z. Li, and X. Wang. A hybrid imputation approach for microarray missing value estimation. BMC Genomics, 16(Suppl 9):S1, 2015. URL https://doi .org/10.1186/1471-216416-s9-s1. [p219]

J. M. Mendel. Uncertainty, fuzzy logic, and signal processing. Signal Processing, 80(6):913-933, 2000. URL https://doi .org/10.1016/s0165-1684(00)00011-6. [p218]

S. Moritz and T. Bartz-Beielstein. imputeTS: Time Series Missing Value Imputation in R. The R Journal, 2017. URL https://journal.r-project.org/archive/2017/RJ-2017-009/index.html. [p219, 220]

J. Muschelli. Matlabr: An Interface for MATLAB Using System Calls, 2016. URL https://CRAN.Rproject. org/package=matlabr. R package version 1.1.3. [p231]

C. D. Nguyen, J. B. Carlin, and K. J. Lee. Diagnosing problems with imputation models using the Kolmogorov-Smirnov test: a simulation study. BMC Medical Research Methodology, 13(1):1, 2013. URL https://doi .org/10.1186/1471-2288-13-144. [p219]

B. Ran, H. Tan, J. Feng, Y. Liu, and W. Wang. Traffic speed data imputation method based on tensor completion. Computational Intelligence and Neuroscience, 2015:22, 2015. URL https://doi .org/10. 1155/2015/364089. [p219]

D. B. Rubin. Inference and missing data. Biometrika, 63(3):581-592, 1976. URL https://doi . org/10. 1093/biomet/63.3.581. [p218, 223]

J. L. Schafer. Analysis of Incomplete Multivariate Data. Chapman and Hall, CRC, Boca Raton, Florida, 1997. ISBN 978-0412040610. [p218]

J. L. Schafer and J. W. Graham. Missing data: Our view of the state of the art. Psychological Methods, 7 (2):147-177, 2002. URL https://doi.org/10.1037//1082-989x.7.2.147. [p218, 224]

R. H. Shumway and D. S. Stoffer. Time Series Analysis and Its Applications: With R Examples. SpringerVerlag, New York, New York, 3rd edition, 2011. [p218]

S. Tak, S. Woo, and H. Yeo. Data-driven imputation method for traffic data in sectional units of road links. IEEE Transactions on Intelligent Transportation Systems, 17(6):1762-1771, 2016. URL https://doi.org/10.1109/tits.2016.2530312. [p219,227]

H. Wickham. Reshaping data with the reshape package. Journal of Statistical Software, 21(12):1-20, 2007. URL https://doi.org/10.18637/jss.v021.i12. [p219]

H. Wickham. Ggplot2: Elegant Graphics for Data Analysis. Springer-Verlag, 2009. ISBN 978-0-387-98140-6. URL http://ggplot2.org. [p219] 
H. Wickham. Tidyr: Easily Tidy Data with spread() and gather() Functions, 2017. URL https:// github.com/tidyverse/tidyr. R package version 0.6.3. [p219]

H. Wickham and R. Francois. Dplyr: A Grammar of Data Manipulation, 2016. URL https: //CRAN. Rproject. org/package=dplyr. R package version 0.7.1. [p219]

C. Yozgatligil, S. Aslan, C. Iyigun, and I. Batmaz. Comparison of missing value imputation methods in time series: The case of Turkish meteorological data. Theoretical and Applied Climatology, 112(1-2): 143-167, 2013. URL https://doi.org/10.1007/s00704-012-0723-x. [p218, 219]

A. Zeileis and G. Grothendieck. Zoo: S3 infrastructure for regular and irregular time series. Journal of Statistical Software, 14(6):1-27, 2005. URL https://doi.org/10.18637/jss.v014.i06. [p219]

X. Zhu, S. Zhang, Z. Jin, Z. Zhang, and Z. Xu. Missing value estimation for mixed-attribute data sets. IEEE Transactions on Knowledge and Data Engineering, 23(1):110-121, 2011. URL https://doi .org/ 10.1109/tkde.2010.99. [p218, 219]

Marcus W Beck

USEPA National Health and Environmental Effects Research Laboratory, Gulf Ecology Division

1 Sabine Island Drive, Gulf Breeze, FL 32651

USA

Current address: Southern California Coastal Water Research Project

3535 Harbor Blvd, Suite 110, Costa Mesa, CA, 92626

USA

marcusb@sccwrp.org

Neeraj Bokde

Visvesvaraya National Institute of Technology, Nagpur

North Ambazari Road, Nagpur

India

neeraj.bokde@students.vnit.ac. in

Gualberto Asencio-Cortés

Universidad Pablo de Olavide

ES-41013, Sevilla

Spain

guaasecor@upo.es

Kishore Kulat

Visvesvaraya National Institute of Technology, Nagpur

North Ambazari Road, Nagpur

India

kdkulat@ece.vnit.ac.in 